\title{
Impacto de un Modelo de Movilización Social sobre la Promoción de la Actividad Física en Afiliados al Sistema de Seguridad Social en Salud
}

\author{
The impact of a social mobilisation model on promoting physical \\ activity amongst people affiliated to the Colombian Social Health \\ Security System
}

Patricia Granada-Echeverri, Carlos D. Zapata-Valencia y José C. Giraldo-Trujillo

Facultad de Ciencias de la Salud, Universidad Tecnológica de Pereira. patriciagranada@utp.edu.co, cazapata@utp.edu.co, jocagirt@utp.edu.co

Recibido 29 Agosto 2007/Enviado para Modificación 19 de Abril 2008/Aceptado 9 Junio 2008

\section{RESUMEN}

Objetivo Desarrollar un Modelo de Movilización Social de promoción de la actividad física como estrategia fundamental de salud.

Métodos Se constituyeron tres poblaciones del Sistema de Seguridad Social en Salud: afiliados al Régimen Contributivo, profesionales de la salud vinculados a EPS, y funcionarios públicos. Se determinó nivel de actividad física, conocimientos, creencias y actitudes, barreras culturales y ambientales, además de aspectos como recomendación por profesionales de la salud y políticas establecidas por funcionarios públicos, y se elaboró el Modelo de intervención.

Resultados La línea de base mostró que la mayoría de afiliados se consideran activos pero en forma inadecuada; reconocen beneficios de la práctica regular pero apenas consideran la posibilidad de realizarla; las principales barreras fueron la falta de autodisciplina, tiempo y necesidad de descansar en su tiempo libre; un alto porcentaje de profesionales de la salud no la recomiendan a sus pacientes y la mayoría de funcionarios públicos manifestaron que está incluida en los planes de desarrollo municipal. Se evaluó el impacto del Modelo identificándose que en las tres poblaciones se generó mayor reconocimiento de la Actividad Física para la salud; modificaron la concepción respecto al tiempo y frecuencia recomendada y se generó mayor cambio de actitud, aunque se mantuvieron las barreras culturales. Los profesionales presentaron cambios relacionados con la percepción de su estado de salud y tendencia a aumentar la recomendación en la consulta médica.

Discusión El modelo de movilización social probó la eficacia de promocionar la actividad física para la prevención de enfermedades crónicas no transmisibles.

Palabras Clave: Actividad física, salud pública, promoción de la salud (fuente: DeCS, BIREME). 


\begin{abstract}
Objective Developing a model of social mobilisation for promoting physical activity as a fundamental health strategy.

Methods Three social health security system groups were considered: people affiliated to the contributive regime, health professionals involved with an EPS and public functionaries. People's levels of physical activity, knowledge, beliefs and attitudes and cultural and environmental barriers were determined; health professionals' recommendations and public policy were also taken into account. An intervention model was then constructed on the foregoing information.

Results The database revealed that most affiliates considered themselves to be active but not in a suitable way; they recognised the benefits of engaging in regular physical activity but they barely considered the possibility of practising it. The main barriers were a lack of self-discipline, lack of time and the need for resting during their free time. A high percentage of health professionals did not recommend it to their patients; most public functionaries stated that physical activity was included in their cities' development strategies. The model's impact was evaluated; it was seen that greater recognition of physical activity was produced in the three groups after applying the model. The concept of recommended time and frequency for physical activity became modified and a greater change in attitudes was produced. However, cultural barriers remained. Health professionals presented changes related to their perception of their state of health and they tended to increase their recommendation for physical activity during medical visits. Discussion The social mobilisation model proved the efficiency of promoting physical activity for preventing non-transmissible chronic diseases.
\end{abstract}

Key Words: Physical activity, public health, health promotion (source: MeSH, NLM).

$\mathrm{E}$ 1 Ministerio de la Protección Social, como respuesta a la alta prevalencia de las Enfermedades Crónicas (EC) asociadas al sedentarismo encontrados en el estudio ENFREC II (1), abrió convocatoria a través de Colciencias en el año 2003 para que en cuatro regiones del País se validara un modelo de movilización social (MMS) para promover la Actividad física (AF) para la salud, dirigida a la población general con énfasis en los afiliados al régimen contributivo (ARC) entre 25-50 años, Empresas Prestadoras de Servicios de Salud (EPS), Instituciones Prestadoras de Servicios (IPS), Administradoras de Riesgos Profesionales (ARP) y demás actores del Sistema de Seguridad Social (SSS). En Risaralda, la UTP y la Gobernación, participaron con el proyecto "Promoción de la Actividad Física para la prevención de las enfermedades crónicas no transmisibles" (PAF/ECNT), que partió de la experiencia acumulada por el Programa Risaralda Activa de Indeportes, diseñado y ejecutado bajo los lineamientos del Programa Agita Sao Paulo (2), reconocido por la OMS como modelo para los países latinoamericanos; y sobre el que la OPS en conjunción con el CDC de los Estados Unidos, están soportando a partir de la Red que promueve la AF en América, "Agita América" (3). 
Estos programas promueven la recomendación del CDC y el American College of Sports Medicine (ACSM), de realizar al menos 30 minutos de AF moderada todos los días, continuos o acumulados. Hasta hace poco se pensaba que sólo los ejercicios intensos de forma continua por más de 30 minutos podían tener impacto positivo sobre la salud; sin embargo, Murphy y cols (4) demostraron beneficios semejantes en protocolos continuos (2 $182 \mathrm{Kcal}$ ) o tres series de 10 minutos (2 $146 \mathrm{Kcal}$ ), sobre la potencia aeróbica, presión arterial, masa corporal y adiposidad y la relación cintura cadera. En este sentido Agita Sao Paulo ha usado un número de estrategias innovadoras y actividades claves para promocionar la AF cotidiana modificando fundamentalmente la actitud de las personas que se clasifican así: Precontemplativas, a quienes el tema de la AF no les interesa y no le dan importancia; Contemplativas, quienes la consideran importante pero no se deciden realizarla regularmente, y Activas, quienes la consideran importante y tienen una AF regular. Entre las estrategias del Programa Agita están: información basada en evidencia científica, mensajes claros y simples, participación de expertos en diferentes áreas relacionadas con el tema en la evaluación de los programas, coaliciones institucionales para llevar a cabo acciones y diseminar el mensaje, soporte técnico y político de gobiernos locales y organizaciones internacionales como OPS, OMS y el CDC, entre otras. El programa es incluyente y se puede ofrecer a individuos y a grupos a toda hora y lugar; el principio de inclusión llega hasta la diversidad cultural y regional. El modelo Mobile que han implementado, administra y balancea los múltiples componentes del modelo ecológico con sus tres partes: interpersonal, factores del medio social y del medio físico, y es exitoso por el soporte multisectorial y las coaliciones estratégicas que su estilo de manejo permite (2).

Risaralda Activa siguiendo estos lineamientos estableció tres metas operativas: (a) Sensibilización (información, educación, planeación para la acción), (b) Fase Activa (participación y gestión pública), (c) Fase de anclaje en las localidades; en su desarrollo ha incidido en 3 poblaciones: adulto mayor, trabajadores y escolares; sin haber evaluado su impacto he implementado la tercera meta. El proyecto PAF/ECNT integra la propuesta, enriquecido con técnicas motivacionales para la adherencia a un programa de ejercicio de Scales (2003) con modelos de movilización aplicados para cambios de comportamiento alimentario, con los aportes conceptuales de la estrategia multinivel deinformación, educación, y comunicación de la Universidad del Valle, y con el modelo macrointencional de Toro (6), que hace énfasis en la creación y fortalecimiento de redes de comunicadores y redes sociales. Si bien Agita Sao Paulo considera en un mismo nivel de jerarquía los tres factores (intrapersonal, medio social y medio físico), consideramos que el modelo del 
PAF/ ECNT debía centrarse en el sujeto, ya que al movilizar representaciones sobre la AF y la salud, creencias, y factores asociados a la cultura, los sujetos sensibilizados, informados, motivados y empoderados podrían influir sobre sus medios social y físico, por lo cual este modelo se llamó "Antrópico", pues se centra en el desarrollo humano para desde allí generar desarrollo social más allá del impacto directo en la disminución de la morbimortalidad por ECNT, que lo hace sostenible al lograr transformaciones estructurales en la actitud y la práctica de las personas.

El propósito general fue diseñar e implementar un MMS que, acorde a las características encontradas, permitiera posicionar la AF como estrategia fundamental en la promoción de la salud y prevención de la enfermedad, es decir como un componente fundamental de las estrategias de Salud Pública.

\section{MATERIALES Y MÉTODOS}

$\mathrm{Al}$ estar dirigido a ARC, el MMS, trabajó el segmento poblacional entre 25-50 años y en las barreras culturales asociadas que se reflejan en la actitud. La validación se desarrolló en tres fases: (a) Línea de base, (b) Diseño e implementación del MMS, (c) Evaluación de impacto. Tres grupos de actores del SSSS fueron constituidos para todo el proceso: Afiliados al régimen contributivo (ARC), profesionales de la salud (PS) y funcionarios públicos (FP). El primero, de 910 personas, se organizó mediante muestreo por cuotas, con los siguientes criterios de inclusión: hombre o mujer, afiliado al régimen contributivo (contribuyente o beneficiario), edad entre 25-50 años. El número de ARC a encuestar se estableció indirectamente (por la imposibilidad de acceder a bases de datos de las EPS por rango de edad), tomando la distribución porcentual de la población general por edad y género conocida por municipio (Censo Dane, 1993) como un factor porcentual (Li) aplicado a los datos de afiliación global del Departamento para el año 2002 (Moreno G, Población compensada de EPS, Informe a la Supersalud, Sept 2001). El segundo grupo se conformó seleccionándose de manera intencional PS entre médicos generales, especialistas y administrativos de las cinco EPS de mayor porcentaje de afiliación, para un total de 57. En el tercer grupo, se incluyeron los 59 sujetos que en el momento desempeñaban cargos de dirección en el sector público (alcaldías, secretarías municipales de salud, educación, deporte y recreación, desarrollo comunitario e infraestructura). Se aplicaron 4 instrumentos validados por Agita Sao Paulo: IPAQ corto; Conocimientos, Actitudes y Prácticas; Barreras Ambientales y Prescripción de la Actividad Física. Las variables fueron: nivel de actividad física, actitud, barreras culturales, barreras ambientales, y prescripción de la AF 
por PS. Se diseñó un instrumento con preguntas semi estructuradas para la recolección de información sobre políticas municipales para FP. Se diseñó la base de datos en Epi-Info 6.0 y los análisis en SPSS 11.5.

Para la evaluación de impacto del MMS, se tomaron como unidad de análisis los grupos de actores del SSSS constituidos para la línea de base. Para los ARC se diseñó un estudio descriptivo, seleccionando y asignando aleatoriamente 250 sujetos de los 910 entrevistados inicialmente, a dos grupos de intervención. Al primero se le realizó intervención mediante medios masivos de comunicación (MMC) y al segundo, se le hizo además intervención intrapersonal individual (PII). 52 de los 57 PS de la línea de base participaron en la fase de aplicación del modelo, distribuidos en los dos grupos de intervención, pero todos tuvieron la misma oferta de capacitación en aspectos relacionados con AF y salud; para la evaluación de impacto se utilizaron los mismos instrumentos de la línea de base adicionando un test de evaluación de MMC. Por otro lado, una evaluación DOFA y el estudio cualitativo para el análisis de impacto de la estrategia de comunicaciones en el nivel comunitario y de política pública fue adelantado mediante grupos focales, entrevistas abiertas, registros audiovisuales y formatos de asistencia y compromiso.

Para el análisis, se compararon los resultados antes y después de la intervención; además de los estadísticos descriptivos, se aplicaron pruebas para muestras independientes ( $t$ de student, chi cuadrado, Mann-Whitney), a fin de establecer si al momento de conformar los grupos existían diferencias en el comportamiento de las variables que pudieran ser atribuibles a efectos aleatorios. Estas comparaciones entre grupos se aplicaron también a los datos obtenidos en la medición post intervención. Para determinar en cada grupo (G1, G2) si los cambios encontrados en la medición final podían atribuirse a alguno de los tipos de intervención, se aplicaron pruebas para muestras relacionadas ( $t$ student, prueba de signos de Wilcoxon).

\section{RESULTADOS}

Línea de base

Nivel de actividad física. El $80 \%$ de ARC entre 25 y 50 años se reconocen como físicamente activos; la mayoría de los que realizaban $\mathrm{AF}$ vigorosa (AFV, 87,4 \%) y AF moderada (AFM, 57,4 \%) lo hacían de forma inadecuada, es decir no cumplen con criterios aceptados internacionalmente sobre intensidad (moderada), frecuencia (5 ó más días de la semana) y duración (30 minutos mínimo continuos o acumulados)); un comportamiento similar fue encontrado en los PS. 
Actitud. La mayoría de la población encuestada (62,3 \% ARC, 56,1 \% PS, 60 \% FP) se encontraba en un nivel de actitud contemplativa sin diferencias por género ni edad, lo cual en términos técnicos significó que estaban apenas considerando asumir la AF como una práctica cotidiana; este nivel estuvo asociado con una práctica inadecuada de la AF. El 56,1 \% de los PS refirieron no promover ó recomendar AF en la consulta.

Barreras culturales. La falta de autodisciplina (32,4 \% en ARC, 75,4 \% en PS, $69,5 \%$ en FP), la falta de tiempo (40,4 \% en ARC, 56,1 \% en PS, 55,9 \% en FP) y la necesidad de descanso pasivo, fueron las barreras culturales más importantes encontradas en estas poblaciones, siendo significativas para la actitud $(\mathrm{p}<0.05)$ más no para la práctica de la AF. Entre el 60 y el 95 \% de ARC estuvieron de acuerdo en que: se puede ir andando fácilmente a tiendas y paradero de buses, existen muchas intersecciones viales en el barrio, y hay pocas calles o carriles para caminar o ir en bicicleta, pocos parques, caminos para pasear y piscinas públicas, aunque no les parece difícil o desagradable transitar por ellas debido al tráfico, ni que caminar o pasear de noche en el barrio sea peligroso; sin embargo, manifestaron no ver muchas personas haciendo ejercicio en el barrio, ni cosas interesantes mientras caminan; sin diferencias significativas para los niveles de actitud ni de práctica de la AF.

El 86 \% de los FP tienen voluntad política para implementar proyectos de investigación en el tema, y aunque se incluyen en los planes de desarrollo, la mayoría son actividades puntuales (deporte o recreación) que no conducen a la formulación de políticas públicas tendientes a promover la AF para la salud. Sólo el 45,8 \% manifestaron realizar campañas de tránsito para facilitar o apoyar la práctica de la AF (cierre de vías para ciclo rutas o ciclo paseos, caminatas, por ejemplo).

\section{Diseño e implementación del Modelo}

A partir de estos resultados, se definieron los siguientes objetivos del MMS: (a) Movilizar positivamente la actitud frente a la práctica de la AF de un porcentaje significativo de ARC, (b) Impactar las barreras que impiden su práctica, (c) Aumentar en los PS su recomendación desde la consulta médica, (d) Incorporar en el colectivo, los conocimientos básicos sobre el tipo, intensidad y duración mínima de AF recomendada para la preservación y/o mantenimiento de la salud, (e) Implementar en las instituciones públicas, privadas y organizaciones sociales, estrategias y mecanismos de participación comunitaria para el diseño y desarrollo de mega eventos de PAF, (f) Posicionar en las EPS, IPS, ARP, ESEs, y 
administraciones públicas locales, el tema de PAF como una prioridad, comprometiéndolas en la implementación de estrategias dirigidas a sus usuarios. Los lineamientos de Agita Sao Paulo y Risaralda Activa, se desarrollaron articulados en las cinco áreas de acción de la Carta de Ottawa (1986): (a) Creación de entornos y capacidad local; (b) Fortalecimiento de redes sociales y de acción comunitaria; (c) Desarrollo de habilidades personales en la población general y en actores claves en la construcción de mensajes y el diseño de estrategias para la promoción de la AF; (d) Reorientación de la promoción de la AF por parte de las IPS; (e) Establecimiento de una política pública saludable a favor de la AF.

El MMS se articula en dos grandes componentes: Educomunicación y Gestión Social. El primero conjuga el enfoque de comunicación masiva, comunicación para el desarrollo y el de educación para el cambio de conducta. Esta integración se propuso tanto en los escenarios de aprendizaje/comunicativos, como en las estrategias dirigidas a individuos y colectividades, donde se privilegió la reflexión en busca de la salud, hasta la protección integral, el cuidado colectivo y la salud pública, combinando técnicas de empoderamiento, abogacía y mercadeo social para posicionar la AF como un bien de dominio público, un deber del Estado y sus instituciones de promoverla, y de los ciudadanos de practicarla. El segundo, de Gestión Social, centra su interés en la movilización de comportamiento del sujeto hacia niveles más activos, orientado teóricamente por el modelo transteorético propuesto por Prochaska (7) en diferentes ámbitos de actuación para la intervención del MMS: intrapersonal, interpersonal, institucional u organizativo, comunitario, política pública. Esta intervención se hizo mediante la estandarización de protocolos, el fortalecimiento y ampliación de redes sociales, la sensibilización a actores claves del SSSS y el posicionamiento político del tema.

Evaluación de impacto del MMS

Con relación a los ARC, en el Comportamiento (Tabla 1) no se observaron cambios significativos ( $>>0.05$ ) en cuanto a percepción del estado de salud, en el paso al que caminan, en el tiempo que permanece sentado por día entre semana, ni en el nivel de AF; se observó diferencia significativa $\mathrm{p}=0.047$ en el tiempo que permanece sentado por día en fin de semana, atribuible a la intervención por MMC. Con relación a los Conocimientos, hubo cambios significativos en ambos tipos de intervención en los consejos para quienes quieren ser sanos ( $\mathrm{p}=0.032$, PII; $\mathrm{p}=0.001, \mathrm{MMC}$ ) y en el tiempo de ejercicio diario necesario para tener buena salud ( $\mathrm{p}=0.007$, PII; $\mathrm{p}=0.023$, MMC); en cuanto al número de días de ejercicio activo para una buena salud, el cambio significativo se dio con relación 
a la intervención intrapersonal ( $\mathrm{p}=0.013$, PII). Respecto a que recomendarían para ser sano, consideran que tener buenas noches de sueño, realizar ejercicio regularmente y no fumar, es importante o muy importante. Se encontró que un $10 \%$ más de personas consideraron muy importante realizar AF regularmente y evitar las comidas grasas, y la AF pasó del cuarto al segundo lugar de importancia. Los que consideraron que es mejor hacer AF en una sola sesión que en periodos cortos pasaron de 59,4 \% a 66,8 \%, y con relación a la intensidad, 57 \% consideran que la mejor actividad es aquella que debe hacer que las personas se sientan ligeramente calientes y respirando con más dificultad que lo normal, lo que denota un incremento en el porcentaje de personas con conocimientos adecuados sobre las características de la AF para la salud. Se observó un incremento de quienes consideraron que caminar es una actividad buena para mejorar la salud. Así mismo, en el grupo de personas con intervención intrapersonal aumentó la proporción (10,3 \%) de quienes consideran que debe realizarse ejercicio activo entre cinco a siete días a la semana. El porcentaje de personas que respondieron que 30 minutos es el tiempo que debe hacerse AF diaria para que sea bueno para la salud pasó de 26,3 \% a 42 \% y quienes respondieron entre 30 y 45 minutos pasó de $31 \%$ a $60 \%$.

Con relación a las creencias, más del $90 \%$, tanto antes como después, estuvieron de acuerdo con las afirmaciones: caminar a diario, caminar o pedalear en lugar de usar el carro y caminar diariamente, son una manera de mantenerse activo. Entre el $60 \%$ y el 95 \% consideraron totalmente importante, muy importante o importante que el ejercicio puede ayudarlos a: relajarse y olvidar preocupaciones, tener alegría, salir, sentir la sensación de realización, sentirse independiente, sentirse alerta, sentirse físicamente en buena forma, aprender cosas nuevas, tener buena apariencia, controlar el peso corporal y mejorar o mantener la salud, sin diferencias según tipo de intervención. Respecto a la Actitud, los niveles Precontemplativo y Contemplativo disminuyeron del 66,8 \% al 58,2 $\%$, aumentando el Activo del 33,2 \% al 41,8 \%. Se aplicó prueba de Wilcoxon para comparar grupos relacionados, encontrándose diferencias significativas de la actitud para el tipo de intervención intrapersonal ( $\mathrm{p}=0.012$ ), más no para la de MMC ( $\mathrm{p}=0.274)$. En cuanto a Barreras Culturales, se observó una disminución porcentual no significativa en todas las barreras que les impiden realizar AF; no obstante, se conservó el orden de frecuencia de presentación. Respecto al impacto de los MMC, el 83 \% refirieron que habían visto o escuchado un mensaje sobre la importancia de la AF para la salud: 44 \% por la televisión regional, 39 \% en la participación en eventos masivos, y $62 \%$ mediante medios impresos. De esta población, el 52 \% dijeron recordar los mensajes relativos a la AF que refieren haber escuchado o visto por algún medio masivo en la siguiente forma: "Por lo 
menos 30 minutos de AF diaria (16,6\%), realizar AF diaria (9,2 \%), hacer ejercicio es saludable (8,1 \%), hacer AF todos los días (5,0 \%) y deporte es salud (4,6 \%)".

Tabla 1. Resultado de pruebas estadísticas (paramétricas y no paramétricas) del impacto del MMS en afiliados al régimen contributivo. Risaralda 2004

\begin{tabular}{|c|c|c|c|c|}
\hline \multirow{2}{*}{\multicolumn{3}{|c|}{ Conceptos y variables impactadas }} & \multicolumn{2}{|c|}{ Evaluación de Impacto } \\
\hline & & & \multirow{2}{*}{$\begin{array}{c}\begin{array}{c}P \| 1 \\
(n=70)\end{array} \\
0.0901\end{array}$} & \multirow{2}{*}{$\begin{array}{c}\begin{array}{c}\text { MMC } \\
(n=180)\end{array} \\
0.335\end{array}$} \\
\hline \multirow[t]{5}{*}{ Comportamierto } & \multicolumn{2}{|c|}{$\begin{array}{l}\text { Percepción del estado de } \\
\text { salud }\end{array}$} & & \\
\hline & \multicolumn{2}{|c|}{ Nivel de AF } & 0.700 & 0.188 \\
\hline & \multicolumn{2}{|c|}{ Paso al que camina } & 0.095 & 0.231 \\
\hline & $\begin{array}{l}\text { Tiempo que } \\
\text { permanece }\end{array}$ & $\begin{array}{l}\text { Entre } \\
\text { semana }\end{array}$ & 0.722 & 0.099 \\
\hline & $\begin{array}{l}\text { sentado por } \\
\text { dia }\end{array}$ & $\begin{array}{l}\text { Fin de } \\
\text { semana }\end{array}$ & 0.194 & $0.047^{*}$ \\
\hline \multirow[t]{3}{*}{ Conocimientos } & \multicolumn{2}{|c|}{$\begin{array}{l}\text { Consejos para quienes } \\
\text { quieren ser sanos }\end{array}$} & $0.032^{*}$ & $0.001^{*}$ \\
\hline & \multicolumn{2}{|c|}{$\begin{array}{l}\text { Números de dlas de } \\
\text { ejercicio activo }\end{array}$} & $0.013^{*}$ & 0.356 \\
\hline & \multicolumn{2}{|c|}{$\begin{array}{l}\text { Tiempo de ejercicio dario } \\
\text { necesario para tener buena } \\
\text { salud }\end{array}$} & $0.007^{*}$ & $0.023^{*}$ \\
\hline Actitud & \multicolumn{2}{|c|}{ Actitud respecto a la AF } & $0.012^{*}$ & 0.274 \\
\hline
\end{tabular}

AF: actividad física; MMS: modelo de movilización social; PII: intervención intrapersonal; MMC: medios masivos de comunicación. *Diferencia significativa, $p<0.05$.

En los PS, respecto al Comportamiento, la percepción de salud excelente disminuyó del $48 \%$ al $27 \%$; el $42 \%$ consideran que su estado de salud es regular; la percepción muy buena y buena se incrementó ligeramente. Hubo diferencias significativas en la percepción de la salud (Tabla 2) en el grupo de PII ( $\mathrm{p}=0.04)$, no así en el de MMC ( $\mathrm{p}=0.190)$. No se encontraron diferencias significativas para el nivel de AF, paso al que caminan y tiempo en el que permanecen sentados por día en un fin de semana. Sobre los Conocimientos, no se encontraron diferencias significativas en los consejos para ser sano y número de días por semana de ejercicio activo. La proporción de quienes respondieron entre cinco a siete días, pasó de 45,8 \% a 62,5\%. Con relación a qué recomendarían para ser sano, aumentó la proporción de los que consideran importante o muy importante tener buenas noches de sueño, evitar engordarse y evitar comidas grasas. No varió la proporción de los que consideran muy importante e importante realizar AF regularmente y ésta se mantuvo en el cuarto lugar de importancia. En cuanto al tiempo diario de ejercicio recomendado, el porcentaje de quienes respondieron 30 minutos pasó de 47,9 \% a 62,7 \%, diferencia significativa en el grupo que recibió PII ( $\mathrm{p}=0.017$ ) (Tabla 2); la proporción de PS que consideran que una sesión diaria es lo recomendable para realizar AF disminuyó, aumentando 
en quienes consideran que ésta puede ser fraccionada en periodos cortos. Con relación a la intensidad, se observó una disminución de los que opinan que el ejercicio debe hacer sentir ligeramente caliente a la persona y hacerla respirar con mayor dificultad. Respecto a las actividades que creen las personas que son buenas para mejorar la salud, se observó un aumento porcentual al considerar el deporte, la caminada, la AF y los ejercicios aeróbicos, como las más recomendadas. Respecto a las Barreras, se tuvo una disminución porcentual no significativa de la mayoría de barreras que les impide realizar AF. Con relación a las Creencias, llama la atención la reducción en un 6,2 \% de quienes concuerdan totalmente en que una caminada es buena para la salud, así como un aumento del 6,4 \% en los que no saben si practicar deporte o hacer ejercicio es más fácil que caminar todo el día. Con relación a la Actitud, no hubo diferencias significativas. En cuanto a la Prescripción de la AF, se pasó en números absolutos de 28 a 46 prescriptores. El número de quienes referían promover la AF regular desde hace más de seis meses pasó de 23 a 32.

Tabla 2. Resultados de pruebas estadísticas (paramétricas y no paramétricas) de la evaluación de impacto del MMS en Profesionales de la Salud (PS). Risaralda 2004

\begin{tabular}{|c|c|c|c|c|}
\hline & \multirow{2}{*}{\multicolumn{2}{|c|}{ Varbable }} & \multicolumn{2}{|c|}{ Evaluación de Impacto } \\
\hline & & & PII $(n=26)$ & MMC $(n=22)$ \\
\hline \multirow[t]{5}{*}{ Comportamiento } & \multicolumn{2}{|c|}{$\begin{array}{l}\text { Percepción del estado de } \\
\text { salud }\end{array}$} & $0.040^{\circ}$ & 0.190 \\
\hline & \multicolumn{2}{|c|}{ Nivel de actividad física } & 0.563 & 0.904 \\
\hline & \multicolumn{2}{|c|}{ Paso al que camina } & 1.0 & 0.705 \\
\hline & $\begin{array}{l}\text { Tiempo que } \\
\text { permanece }\end{array}$ & $\begin{array}{l}\text { Entre } \\
\text { semana }\end{array}$ & 0.172 & $0.004^{*}$ \\
\hline & sentado por dia & $\begin{array}{l}\text { Fin de } \\
\text { semana }\end{array}$ & 0.184 & 0.360 \\
\hline \multirow[t]{3}{*}{ Conocimientos } & \multicolumn{2}{|c|}{$\begin{array}{l}\text { Consejos para quienes } \\
\text { quiemen ser sanos }\end{array}$} & 0.446 & 0.883 \\
\hline & \multicolumn{2}{|c|}{$\begin{array}{l}\text { Numeros de dias de ejercicio } \\
\text { activo }\end{array}$} & 0.318 & 0.924 \\
\hline & \multicolumn{2}{|c|}{$\begin{array}{l}\text { Tiempo de ejercicio diario } \\
\text { necesario para tener buena } \\
\text { saud }\end{array}$} & $0.017^{*}$ & 0.140 \\
\hline Actitud & \multicolumn{2}{|c|}{ Actitud respecto a la $\mathrm{AF}$} & 0.285 & 0,705 \\
\hline
\end{tabular}

AF: actividad física; MMS: modelo de movilización social; PII: intervención intrapersonal; MMC: medios masivos de comunicación. *Diferencia significativa, $\mathrm{p}<0.05$

\section{DISCUSIÓN}

El principal factor de riesgo en los ARC y en PS en Risaralda es no tener conciencia del riesgo y la principal barrera es la actitud para asumir el cambio de comportamiento saludable. Esto posiblemente se debe al hecho de que las personas pueden ser activas en algún momento por condiciones del medio externo y dejan de serlo al cambiar ese contexto. La teoría acumulada sobre intervenciones para el 
cambio de comportamiento señala la importancia de los MMC para sensibilizar e informar pero no para un cambio de actitud, Risaralda, contraria a las que operan para el modelo Mobile de Agita Sao Paulo diseñado para grandes ciudades con más de 7 millones de habitantes.

La evidencia del impacto del modelo Antrópico en sus componentes puede ser comparada frente a la evidencia de las medidas efectivas para incrementar la AF realizada por el CDC mediante revisión bibliográfica (8).

De acuerdo a los resultados, es recomendable integrar en los procesos de difusión masiva, a medianos y pequeños medios de comunicación de radio y TV e integrarlos en el trabajo en redes sociales y en procesos de planeación participativa con otros actores de la comunidad $(9,10)$. Se recomiendan impresos y plegables institucionales con poca información en los eventos masivos (8), y con amplia información en los procesos personalizados, mediados por un monitor o experto y respaldados con una guía de reflexión y análisis. Cobran importancia los materiales elaborados por las comunidades con sus propios recursos y simbologías, ajustando el contenido de los productos comunicativos precisando pautas de comportamiento que indiquen el qué, cuánto, cómo, donde, por qué y el para qué, en mensajes sencillos y cortos. Aunque el CDC clasifica las campañas de MMC con evidencia insuficiente, el proyecto PAF/ECNT encontró evidencia estadística del impacto de estas frente a la "disminución del tiempo que permanecen sentados el fin de semana, recomendaciones para quienes quieren ser sanos y número de días necesarios para que la AF sea buena para la salud", aspectos que lograron ser movilizados significativamente.

El componente de gestión social se valida con la PII en ARC para el cambio de actitud y de algunas barreras relacionadas con los conocimientos, más no así en los PS cuya actitud se movilizó negativamente afectando apenas su autopercepción en el nivel de salud y delimitando los conceptos centrales de la recomendación de la AF para la salud. Aunque no hubo cambios significativos en las personas que pasaron a ser activas, se logró que ellos se interesaran en conocer los parámetros para realizar AF en forma adecuada; esto se puede notar en los cambios en los niveles de la intensidad de la AF realizada. Es importante señalar el impacto que tiene para la sociedad en general y para el SSSS, la intervención dirigida a PS, mediante programas individuales de cambios de conducta (estrategia fuertemente recomendada por el CDC) para el cumplimiento de las funciones esenciales de promoción de la salud y salud pública (11). No hay evidencia suficiente sobre intervenciones para el cambio de comportamiento con relación a la promoción de la AF en el consultorio, mientras que existe para aulas de clase, centros 
educacionales y lugares de trabajo (10). En la intervención masiva, se concluye como altamente recomendables actividades como caminatas, stands y carreras de aventura; para directivos del SSSS se recomiendan talleres y seminarios, no así para los PS quienes asisten en muy bajo porcentaje, posiblemente por el control excesivo de tiempo por parte de los empleadores.

El problema de la educación continua de PS del SSSS, requiere del esfuerzo concertado entre universidades, EPS, sector público y demás actores del sistema, para que se prioricen temas nodales de formación que impactan múltiples niveles de la salud, como acontece con la AF vista como un indicador positivo en salud. Queda el reto de diseñar procesos de educación continuada a los PS, que conjuguen sus variables intrapersonales como actitud, motivación, e interés, con las variables laborales que les limita el acceso a estos espacios formativos.

A pesar de ser altamente recomendadas las acciones de información a los usuarios de EPS, no se evidenció un interés significativo en éstas por implementar programas de promoción y prevención en este campo. Las ARS consideran que su ámbito de actuación se circunscribe a recordar a los empleadores las normas de seguridad en el trabajo, obviando la obligatoriedad de complementar y orientar programas de promoción y prevención dentro del plan de salud ocupacional de cada empresa. La realización de talleres en pequeños grupos comunitarios para el diseño, planeación y ejecución de las caminatas, favoreció la participación ciudadana y fue relevante para el intercambio de información, posicionando la AF como un bien público. Esta estrategia aunque puede corresponder a actividades de base comunitaria según la clasificación del CDC (8) no está contemplada dentro de sus recomendaciones, de manera diferenciada. La Red de Comunicadores Comunitarios de la AF de Risaralda, quedó conformada por representantes de instituciones de salud, educación, cultura, deportes, organizaciones y grupos comunitarios, defensa civil, bomberos, alcaldía, policía, medios de comunicación comunitarios, entre otras. Los contactos previos con la Red Colombiana de Actividad Física, la Asociación de Medicina del Deporte de Colombia(AMEDCO), la Red Panamericana de Actividad Física (RAFA-PANA) y Agita Mundo, así como la asesoría de Agita Sao Paulo, fortalecieron los vínculos durante el presente proyecto *

Agradecimientos. Trabajo realizado en el Marco del Proyecto "Promoción de la Actividad Física para la prevención de enfermedades crónicas no transmisibles", patrocinado por Colciencias y el Ministerio de la Protección Social. 


\section{REFERENCIAS}

1. Ministerio de Salud. II estudio nacional de factores de riesgo de Enfermedades Crónicas ENFREC II. Serie Documentos técnicos Tomo III; 1999. pp. 41-62.

2. Matsudo V, Matsudo S. Programa Agita São Paulo. Centro Estudos do Laboratorio de Aptidão Física de São Caetano do Sul (Celafiscs); 2002.

3. Matsudo V, Matsudo S, Andrade D, Araujo T, Andrade E, Oliveira L, et al. Promotion of physical activity in a developing country: The Agita São Paulo experience. Public Health Nutrition 2002; 5(1): 253-261.

4. Murphy M, Hardman A. Training effects of short and long bouts of brisk walking in sedentary women. Med Sci Sports Excer 1998; 30(1): 152-157.

5. Mejía C, Martínez JW, Ospina J. Conocimientos y nivel de Actividad Física desarrollada en la población Risaraldense. Pereira, JM Calle ed Septiembre de 2002.

6. Toro B, Rodriguez MC. La comunicación y la movilización social en la construcción de bienes públicos. Banco Interamericano de Desarrollo (BID). Bogotá, Colombia; Enero 2001.

7. Prochaska JO, Marcus BH. The transtheoretical model: Applications to exercise. Dishman RK, ed. Advances in exercise adherence. Champaing, Illinois: Human kinetics; 1994. pp.161-180.

8. US Department of Health and Human Services. Physical Activity Evaluation Handbook. Atlanta GA: US Department of Health and human services. Centers for Disease Control and Prevention; 2002.

9. Vio del Río F, Salinas CJ. Guía para una vida activa. Santiago de Chile. Andros impresores; 2003.

10. Sharpe P. Investigación participativa para promover la Actividad Física en South Carolina: el papel del mercadeo social para fomentar el cambio de comportamientos. Extramural prevention research program. EPRP, CDC; 2004.

11. Muñoz F, Salinas J. Promoción de la salud como política de Estado. En: Bases, prioridades y desafíos de la Promoción de la Salud. Santiago de Chile. Andros Impresores; 2004. 